\title{
Supplementary Table 2.
}

List of deregulated mature miRNAs in FTO knockdown cells. We detected 249 miRNAs with significantly increased levels (>1.5-fold change) (a) and 35 miRNAs with significantly decreased levels (b). Means and standard deviations of three compared pairs of scrambled and FTO specific siRNA treated cells are given.

\section{a. Upregulated miRNAs in FTO deficient cells.}

\begin{tabular}{|c|c|c|c|}
\hline & Feature & Mean & SD \\
\hline 1 & hsa-miR-6505-5p & 9.83 & 4.61 \\
\hline 2 & hsa-miR-651-5p & 6.49 & 1.61 \\
\hline 3 & hsa-miR-493-5p & 3.99 & 0.98 \\
\hline 4 & hsa-miR-6739-3p & 3.84 & 1.03 \\
\hline 5 & hsa-miR-221-3p & 2.79 & 0.40 \\
\hline 6 & hsa-miR-4492 & 2.62 & 0.43 \\
\hline 7 & hsa-miR-5589-5p & 2.62 & 0.51 \\
\hline 8 & hsa-miR-1245b-5p & 2.53 & 0.51 \\
\hline 9 & hsa-miR-6763-5p & 2.42 & 0.27 \\
\hline 10 & hsa-miR-1246 & 2.41 & 0.49 \\
\hline 11 & hsa-miR-23c & 2.37 & 0.23 \\
\hline 12 & hsa-miR-4489 & 2.34 & 0.97 \\
\hline 13 & hsa-let-7b-5p & 2.31 & 0.17 \\
\hline 14 & hsa-miR-6869-5p & 2.30 & 0.80 \\
\hline 15 & hsa-miR-7161-5p & 2.29 & 0.98 \\
\hline 16 & hsa-miR-3681-5p & 2.28 & 0.36 \\
\hline 17 & hsa-miR-1324 & 2.27 & 0.09 \\
\hline 18 & hsa-miR-663a & 2.18 & 0.50 \\
\hline 19 & hsa-miR-30a-3p & 2.17 & 0.15 \\
\hline 20 & hsa-miR-6795-3p & 2.16 & 0.67 \\
\hline 21 & hsa-miR-4664-5p & 2.15 & 0.69 \\
\hline 22 & hsa-miR-6506-5p & 2.13 & 0.32 \\
\hline 23 & hsa-miR-1258 & 2.11 & 0.44 \\
\hline 24 & hsa-miR-5787 & 2.11 & 0.32 \\
\hline 25 & hsa-miR-6758-5p & 2.11 & 0.23 \\
\hline 26 & hsa-miR-127-5p & 2.10 & 0.67 \\
\hline 27 & hsa-miR-5682 & 2.09 & 0.20 \\
\hline 28 & hsa-miR-6513-3p & 2.07 & 0.54 \\
\hline 29 & hsa-miR-1257 & 2.04 & 0.25 \\
\hline 30 & hsa-miR-646 & 2.03 & 0.68 \\
\hline 31 & hsa-miR-635 & 2.02 & 0.44 \\
\hline 32 & hsa-miR-302a-5p & 2.02 & 0.03 \\
\hline 33 & hsa-miR-6090 & 2.02 & 0.47 \\
\hline
\end{tabular}




\begin{tabular}{|c|c|c|c|}
\hline 34 & hsa-miR-3616-3p & 2.01 & 0.43 \\
\hline 35 & hsa-miR-4502 & 1.99 & 0.38 \\
\hline 36 & hsa-miR-580-3p & 1.99 & 0.13 \\
\hline 37 & hsa-miR-573 & 1.98 & 0.06 \\
\hline 38 & hsa-miR-4763-3p & 1.98 & 0.18 \\
\hline 39 & hsa-miR-144-3p & 1.97 & 0.43 \\
\hline 40 & hsa-miR-3609 & 1.97 & 0.24 \\
\hline 41 & hsa-miR-5088-3p & 1.97 & 0.70 \\
\hline 42 & hsa-miR-5089-5p & 1.96 & 0.45 \\
\hline 43 & hsa-miR-924 & 1.95 & 0.38 \\
\hline 44 & hsa-miR-4747-3p & 1.94 & 0.22 \\
\hline 45 & hsa-miR-6088 & 1.93 & 0.45 \\
\hline 46 & hsa-miR-152-3p & 1.93 & 0.16 \\
\hline 47 & hsa-miR-200b-5p & 1.93 & 0.35 \\
\hline 48 & hsa-miR-4791 & 1.92 & 0.25 \\
\hline 49 & hsa-miR-711 & 1.92 & 0.83 \\
\hline 50 & hsa-miR-4483 & 1.92 & 0.54 \\
\hline 51 & hsa-miR-1182 & 1.92 & 0.73 \\
\hline 52 & hsa-miR-4423-5p & 1.92 & 0.81 \\
\hline 53 & hsa-miR-1275 & 1.92 & 0.41 \\
\hline 54 & hsa-let-7f-2-3p & 1.89 & 0.52 \\
\hline 55 & hsa-miR-8082 & 1.88 & 0.30 \\
\hline 56 & hsa-miR-5089-3p & 1.88 & 0.26 \\
\hline 57 & hsa-miR-1203 & 1.87 & 0.60 \\
\hline 58 & hsa-miR-6791-5p & 1.87 & 0.78 \\
\hline 59 & hsa-miR-668-5p & 1.86 & 0.70 \\
\hline 60 & hsa-miR-644a & 1.85 & 0.38 \\
\hline 61 & hsa-miR-506-3p & 1.85 & 0.23 \\
\hline 62 & hsa-miR-30e-3p & 1.85 & 0.04 \\
\hline 63 & hsa-miR-382-5p & 1.85 & 0.80 \\
\hline 64 & hsa-miR-3607-5p & 1.84 & 0.43 \\
\hline 65 & hsa-miR-485-5p & 1.84 & 0.34 \\
\hline 66 & hsa-miR-4475 & 1.84 & 0.32 \\
\hline 67 & hsa-miR-4731-5p & 1.82 & 0.82 \\
\hline 68 & hsa-miR-3617-3p & 1.82 & 0.47 \\
\hline 69 & hsa-miR-3920 & 1.82 & 0.20 \\
\hline 70 & hsa-miR-1238-5p & 1.81 & 0.56 \\
\hline 71 & hsa-miR-656-5p & 1.80 & 0.76 \\
\hline 72 & hsa-miR-548f-3p & 1.77 & 0.30 \\
\hline 73 & hsa-miR-4485 & 1.77 & 0.30 \\
\hline 74 & hsa-miR-605-3p & 1.77 & 0.17 \\
\hline 75 & hsa-miR-8078 & 1.76 & 0.29 \\
\hline 76 & hsa-miR-3195 & 1.76 & 0.38 \\
\hline 77 & hsa-miR-6806-5p & 1.76 & 0.35 \\
\hline
\end{tabular}




\begin{tabular}{|c|c|c|c|}
\hline 78 & hsa-miR-4517 & 1.75 & 0.12 \\
\hline 79 & hsa-miR-4444 & 1.75 & 0.34 \\
\hline 80 & hsa-miR-3680-3p & 1.75 & 0.40 \\
\hline 81 & hsa-miR-4677-5p & 1.75 & 0.28 \\
\hline 82 & hsa-miR-4634 & 1.74 & 0.40 \\
\hline 83 & hsa-miR-19a-5p & 1.74 & 0.22 \\
\hline 84 & hsa-miR-4256 & 1.74 & 0.13 \\
\hline 85 & hsa-miR-3943 & 1.74 & 0.53 \\
\hline 86 & hsa-miR-4701-5p & 1.73 & 0.40 \\
\hline 87 & hsa-miR-4521 & 1.73 & 0.43 \\
\hline 88 & hsa-miR-4707-5p & 1.73 & 0.41 \\
\hline 89 & hsa-miR-6759-5p & 1.73 & 0.61 \\
\hline 90 & hsa-miR-5579-3p & 1.72 & 0.30 \\
\hline 91 & hsa-miR-4670-5p & 1.72 & 0.27 \\
\hline 92 & hsa-miR-5703 & 1.72 & 0.63 \\
\hline 93 & hsa-miR-1266-3p & 1.72 & 0.23 \\
\hline 94 & hsa-miR-4515 & 1.72 & 0.26 \\
\hline 95 & hsa-miR-4717-3p & 1.72 & 0.52 \\
\hline 96 & hsa-miR-6125 & 1.72 & 0.45 \\
\hline 97 & hsa-miR-6877-3p & 1.72 & 0.40 \\
\hline 98 & hsa-miR-6133 & 1.71 & 0.46 \\
\hline 99 & hsa-miR-363-5p & 1.71 & 0.27 \\
\hline 100 & hsa-miR-4488 & 1.71 & 0.14 \\
\hline 101 & hsa-miR-1261 & 1.70 & 0.10 \\
\hline 102 & hsa-miR-145-3p & 1.70 & 0.20 \\
\hline 103 & hsa-miR-5195-3p & 1.70 & 0.43 \\
\hline 104 & hsa-miR-4722-3p & 1.70 & 0.46 \\
\hline 105 & hsa-miR-1181 & 1.70 & 0.57 \\
\hline 106 & hsa-miR-1237-5p & 1.70 & 0.35 \\
\hline 107 & hsa-miR-3622b-3p & 1.70 & 0.14 \\
\hline 108 & hsa-miR-6789-5p & 1.70 & 0.28 \\
\hline 109 & hsa-let-7i-5p & 1.70 & 0.35 \\
\hline 110 & hsa-miR-337-3p & 1.69 & 0.37 \\
\hline 111 & hsa-miR-6780b-5p & 1.69 & 0.34 \\
\hline 112 & hsa-miR-566 & 1.69 & 0.23 \\
\hline 113 & hsa-miR-499a-5p & 1.68 & 0.28 \\
\hline 114 & hsa-miR-3940-5p & 1.68 & 0.21 \\
\hline 115 & hsa-miR-3192-3p & 1.68 & 0.61 \\
\hline 116 & hsa-miR-513c-3p & 1.68 & 0.48 \\
\hline 117 & hsa-miR-4711-3p & 1.68 & 0.14 \\
\hline 118 & hsa-miR-8069 & 1.68 & 0.48 \\
\hline 119 & hsa-miR-631 & 1.67 & 0.45 \\
\hline 120 & hsa-miR-3942-5p & 1.67 & 0.31 \\
\hline 121 & hsa-miR-3150b-3p & 1.67 & 0.17 \\
\hline
\end{tabular}




\begin{tabular}{|c|c|c|c|}
\hline 122 & hsa-miR-3928-5p & 1.67 & 0.02 \\
\hline 123 & hsa-miR-6792-5p & 1.66 & 0.34 \\
\hline 124 & hsa-miR-1263 & 1.66 & 0.19 \\
\hline 125 & hsa-miR-587 & 1.66 & 0.20 \\
\hline 126 & hsa-miR-1272 & 1.66 & 0.05 \\
\hline 127 & hsa-miR-6822-5p & 1.66 & 0.57 \\
\hline 128 & hsa-miR-3960 & 1.66 & 0.43 \\
\hline 129 & hsa-miR-1250-5p & 1.66 & 0.20 \\
\hline 130 & hsa-miR-8066 & 1.65 & 0.39 \\
\hline 131 & hsa-miR-298 & 1.65 & 0.20 \\
\hline 132 & hsa-miR-136-5p & 1.65 & 0.32 \\
\hline 133 & hsa-miR-4535 & 1.65 & 0.34 \\
\hline 134 & hsa-miR-30b-3p & 1.65 & 0.32 \\
\hline 135 & hsa-miR-8058 & 1.64 & 0.06 \\
\hline 136 & hsa-miR-3934-5p & 1.64 & 0.39 \\
\hline 137 & hsa-miR-6722-3p & 1.64 & 0.28 \\
\hline 138 & hsa-miR-6836-3p & 1.64 & 0.11 \\
\hline 139 & hsa-miR-3652 & 1.63 & 0.40 \\
\hline 140 & hsa-miR-653-3p & 1.63 & 0.29 \\
\hline 141 & hsa-miR-3682-3p & 1.63 & 0.20 \\
\hline 142 & hsa-miR-5694 & 1.63 & 0.26 \\
\hline 143 & hsa-miR-3944-3p & 1.63 & 0.17 \\
\hline 144 & hsa-miR-4999-5p & 1.63 & 0.24 \\
\hline 145 & hsa-miR-431-3p & 1.63 & 0.42 \\
\hline 146 & hsa-miR-6503-5p & 1.63 & 0.52 \\
\hline 147 & hsa-miR-4745-3p & 1.62 & 0.38 \\
\hline 148 & hsa-miR-4699-3p & 1.62 & 0.24 \\
\hline 149 & hsa-miR-3662 & 1.62 & 0.05 \\
\hline 150 & hsa-miR-4652-5p & 1.62 & 0.45 \\
\hline 151 & hsa-miR-26b-5p & 1.62 & 0.32 \\
\hline 152 & hsa-miR-5001-5p & 1.62 & 0.39 \\
\hline 153 & hsa-miR-6719-3p & 1.62 & 0.23 \\
\hline 154 & hsa-miR-29b-2-5p & 1.62 & 0.34 \\
\hline 155 & hsa-miR-4417 & 1.61 & 0.25 \\
\hline 156 & hsa-miR-5685 & 1.61 & 0.17 \\
\hline 157 & hsa-miR-521 & 1.61 & 0.50 \\
\hline 158 & hsa-miR-137 & 1.61 & 0.27 \\
\hline 159 & hsa-miR-1908-3p & 1.61 & 0.36 \\
\hline 160 & hsa-miR-6863 & 1.61 & 0.23 \\
\hline 161 & hsa-miR-6827-5p & 1.60 & 0.41 \\
\hline 162 & hsa-miR-3614-5p & 1.60 & 0.16 \\
\hline 163 & hsa-miR-3182 & 1.60 & 0.10 \\
\hline 164 & hsa-miR-4729 & 1.60 & 0.39 \\
\hline 165 & hsa-miR-6726-3p & 1.60 & 0.14 \\
\hline
\end{tabular}




\begin{tabular}{|c|c|c|c|}
\hline 166 & hsa-miR-508-5p & 1.60 & 0.35 \\
\hline 167 & hsa-miR-4504 & 1.59 & 0.40 \\
\hline 168 & hsa-miR-4792 & 1.59 & 0.13 \\
\hline 169 & hsa-miR-3163 & 1.59 & 0.36 \\
\hline 170 & hsa-miR-4667-5p & 1.59 & 0.34 \\
\hline 171 & hsa-miR-1184 & 1.59 & 0.52 \\
\hline 172 & hsa-miR-6823-5p & 1.58 & 0.29 \\
\hline 173 & hsa-miR-197-5p & 1.58 & 0.28 \\
\hline 174 & hsa-miR-6793-5p & 1.58 & 0.18 \\
\hline 175 & hsa-miR-98-3p & 1.58 & 0.10 \\
\hline 176 & hsa-miR-4747-5p & 1.58 & 0.40 \\
\hline 177 & hsa-miR-567 & 1.58 & 0.14 \\
\hline 178 & hsa-miR-6089 & 1.57 & 0.07 \\
\hline 179 & hsa-miR-4778-5p & 1.57 & 0.41 \\
\hline 180 & hsa-miR-499b-5p & 1.57 & 0.47 \\
\hline 181 & hsa-miR-1256 & 1.57 & 0.14 \\
\hline 182 & hsa-miR-4711-5p & 1.57 & 0.16 \\
\hline 183 & hsa-miR-6739-5p & 1.57 & 0.47 \\
\hline 184 & hsa-miR-8072 & 1.57 & 0.13 \\
\hline 185 & hsa-miR-7704 & 1.56 & 0.26 \\
\hline 186 & hsa-miR-4497 & 1.56 & 0.16 \\
\hline 187 & hsa-miR-6761-5p & 1.56 & 0.32 \\
\hline 188 & hsa-miR-4737 & 1.56 & 0.17 \\
\hline 189 & hsa-miR-2115-3p & 1.55 & 0.12 \\
\hline 190 & hsa-miR-1910-3p & 1.55 & 0.13 \\
\hline 191 & hsa-miR-4281 & 1.55 & 0.18 \\
\hline 192 & hsa-miR-601 & 1.55 & 0.40 \\
\hline 193 & hsa-miR-606 & 1.55 & 0.18 \\
\hline 194 & hsa-miR-3131 & 1.55 & 0.41 \\
\hline 195 & hsa-miR-4734 & 1.55 & 0.35 \\
\hline 196 & hsa-miR-4766-5p & 1.55 & 0.11 \\
\hline 197 & hsa-miR-4420 & 1.55 & 0.27 \\
\hline 198 & hsa-miR-3196 & 1.55 & 0.26 \\
\hline 199 & hsa-miR-4718 & 1.55 & 0.24 \\
\hline 200 & hsa-miR-4524a-3p & 1.55 & 0.37 \\
\hline 201 & hsa-miR-4767 & 1.55 & 0.33 \\
\hline 202 & hsa-miR-4650-3p & 1.55 & 0.32 \\
\hline 203 & hsa-miR-4739 & 1.55 & 0.34 \\
\hline 204 & hsa-miR-4503 & 1.55 & 0.11 \\
\hline 205 & hsa-miR-6132 & 1.55 & 0.27 \\
\hline 206 & hsa-miR-6845-3p & 1.54 & 0.35 \\
\hline 207 & hsa-miR-6079 & 1.54 & 0.06 \\
\hline 208 & hsa-miR-8073 & 1.54 & 0.21 \\
\hline 209 & hsa-miR-1289 & 1.54 & 0.28 \\
\hline
\end{tabular}




\begin{tabular}{|c|c|c|c|}
\hline 210 & hsa-miR-3137 & 1.54 & 0.09 \\
\hline 211 & hsa-miR-6816-3p & 1.54 & 0.36 \\
\hline 212 & hsa-miR-203b-3p & 1.54 & 0.22 \\
\hline 213 & hsa-miR-4292 & 1.54 & 0.43 \\
\hline 214 & hsa-miR-6729-5p & 1.54 & 0.20 \\
\hline 215 & hsa-miR-6499-3p & 1.54 & 0.10 \\
\hline 216 & hsa-miR-6869-3p & 1.54 & 0.35 \\
\hline 217 & hsa-miR-5707 & 1.53 & 0.34 \\
\hline 218 & hsa-miR-7703 & 1.53 & 0.03 \\
\hline 219 & hsa-miR-541-5p & 1.53 & 0.45 \\
\hline 220 & hsa-miR-6790-3p & 1.53 & 0.06 \\
\hline 221 & hsa-miR-200c-3p & 1.52 & 0.22 \\
\hline 222 & hsa-miR-410-5p & 1.52 & 0.28 \\
\hline 223 & hsa-miR-7705 & 1.52 & 0.16 \\
\hline 224 & hsa-miR-5004-3p & 1.52 & 0.29 \\
\hline 225 & hsa-miR-7974 & 1.52 & 0.17 \\
\hline 226 & hsa-miR-3651 & 1.52 & 0.30 \\
\hline 227 & hsa-miR-6788-5p & 1.52 & 0.38 \\
\hline 228 & hsa-miR-6515-5p & 1.52 & 0.34 \\
\hline 229 & hsa-miR-372-3p & 1.52 & 0.18 \\
\hline 230 & hsa-miR-6876-3p & 1.52 & 0.35 \\
\hline 231 & hsa-miR-1343-5p & 1.52 & 0.36 \\
\hline 232 & hsa-miR-1231 & 1.52 & 0.22 \\
\hline 233 & hsa-miR-4524b-3p & 1.52 & 0.49 \\
\hline 234 & hsa-miR-762 & 1.52 & 0.22 \\
\hline 235 & hsa-miR-3692-3p & 1.52 & 0.15 \\
\hline 236 & hsa-miR-3923 & 1.52 & 0.14 \\
\hline 237 & hsa-miR-33b-3p & 1.52 & 0.33 \\
\hline 238 & hsa-miR-3145-3p & 1.51 & 0.35 \\
\hline 239 & hsa-miR-643 & 1.51 & 0.16 \\
\hline 240 & hsa-miR-3620-5p & 1.51 & 0.33 \\
\hline 241 & hsa-miR-6878-5p & 1.51 & 0.28 \\
\hline 242 & hsa-miR-559 & 1.51 & 0.22 \\
\hline 243 & hsa-miR-3613-5p & 1.50 & 0.19 \\
\hline 244 & hsa-miR-4467 & 1.50 & 0.30 \\
\hline 245 & hsa-miR-7977 & 1.50 & 0.18 \\
\hline 246 & hsa-miR-7846-3p & 1.50 & 0.13 \\
\hline 247 & hsa-miR-6501-3p & 1.50 & 0.32 \\
\hline 248 & hsa-miR-4533 & 1.50 & 0.30 \\
\hline 249 & hsa-miR-624-5p & 1.50 & 0.32 \\
\hline
\end{tabular}




\section{b. Downregulated miRNAs in FTO deficient cells.}

\begin{tabular}{|c|c|c|c|}
\hline & Feature & Mean & SD \\
\hline 1 & hsa-miR-22-3p & 0.28 & 0.02 \\
\hline 2 & hsa-miR-196a-5p & 0.35 & 0.06 \\
\hline 3 & hsa-miR-7-5p & 0.40 & 0.23 \\
\hline 4 & hsa-miR-769-5p & 0.49 & 0.07 \\
\hline 5 & hsa-miR-92a-1-5p & 0.52 & 0.17 \\
\hline 6 & hsa-miR-671-5p & 0.55 & 0.15 \\
\hline 7 & hsa-miR-6769a-3p & 0.56 & 0.28 \\
\hline 8 & hsa-miR-27b-3p & 0.56 & 0.07 \\
\hline 9 & hsa-miR-215-5p & 0.61 & 0.07 \\
\hline 10 & hsa-miR-1180-3p & 0.63 & 0.07 \\
\hline 11 & hsa-miR-576-5p & 0.63 & 0.02 \\
\hline 12 & hsa-miR-28-3p & 0.65 & 0.03 \\
\hline 13 & hsa-miR-25-3p & 0.66 & 0.04 \\
\hline 14 & hsa-miR-449c-5p & 0.66 & 0.05 \\
\hline 15 & hsa-miR-641 & 0.67 & 0.03 \\
\hline 16 & hsa-miR-330-3p & 0.67 & 0.06 \\
\hline 17 & hsa-miR-1286 & 0.68 & 0.07 \\
\hline 18 & hsa-miR-561-5p & 0.69 & 0.11 \\
\hline 19 & hsa-miR-9-5p & 0.69 & 0.11 \\
\hline 20 & hsa-miR-218-5p & 0.69 & 0.12 \\
\hline 21 & hsa-miR-195-5p & 0.70 & 0.11 \\
\hline 22 & hsa-miR-148a-5p & 0.71 & 0.10 \\
\hline 23 & hsa-miR-16-5p & 0.71 & 0.04 \\
\hline 24 & hsa-miR-31-5p & 0.71 & 0.08 \\
\hline 25 & hsa-miR-1910-5p & 0.72 & 0.17 \\
\hline 26 & hsa-miR-3176 & 0.72 & 0.12 \\
\hline 27 & hsa-miR-4661-3p & 0.73 & 0.19 \\
\hline 28 & hsa-miR-877-5p & 0.73 & 0.01 \\
\hline 29 & hsa-miR-148b-5p & 0.73 & 0.10 \\
\hline 30 & hsa-miR-423-5p & 0.74 & 0.04 \\
\hline 31 & hsa-miR-941 & 0.74 & 0.03 \\
\hline 32 & hsa-miR-181b-5p & 0.74 & 0.11 \\
\hline 33 & hsa-miR-548b-5p & 0.75 & 0.08 \\
\hline 34 & hsa-miR-30e-5p & 0.75 & 0.19 \\
\hline 35 & hsa-miR-21-5p & 0.76 & 0.18 \\
\hline
\end{tabular}

